\title{
LA EPISTEMOLOGÍA RACIONALISTA CRÍTICA DE POPPER Y LA EPISTEMOLOGÍA DEL SUR DE BOAVENTURA DE SOUZA SANTOS. COMPARACIÓN CRÍTICA.
}

\author{
Amancio VÁZQUEZ ${ }^{1}$
}

\section{Resumen}

El artículo realiza una comparación crítica entre dos posturas epistemológicas claramente opuestas, las que al fundamentarse en cosmovisiones antagónicas entran en conflicto. Por un lado, la "Perspectiva Hegemónica" que encuentra su modalidad más acabada en la obra de Karl Popper, y por el otro, la "Perspectiva Alternativa" que se expresa en la obra de Boaventura de Souza Santos. El texto sostiene que el dominio en el que se dirimen sus fundamentos cognitivos es en mayor medida de orden político que epistemológico.

Palabras clave: Epistemología - Karl Popper- Boaventura de Souza Santos-"Perspectiva Hegemónica" - "Perspectiva Alternativa" -

\section{Résumé}

L'article fait une comparaison critique entre deux regards épistémologiques clairement confrontes. Lorsqu'ils sont fondés sur des perspectives anatagonistes du monde, le conflit parmi eux apparaît. D’une part, la «perspective hégémonique » qui trouve sa pleine forme dans l'œuvre de Karl Popper, et d'autre part, la « perspective alternative » qui s'exprime dans le travail de Boaventura de Souza Santos. Le texte soutient que le domaine dans lequel s'opposent les fondements cognitifs est plus politique qu'épistémologique.

Mots-clés: épistémologie - KarlPopper -Boaventura de Souza Santos- Perspective hégémonique - Perspective alternative

\section{Abstract \\ The article performs a critical comparison between two clearly opposite epistemological approaches which, being based on antagonistic worldviews, collide: on one hand, the \\ ${ }^{1}$ Licenciado en Ciencia Política. Docente Universidad Nacional del Litoral y Universidad Nacional de Rosario, Argentina. amanciovazquez@hotmail.com}


"hegemonic perspective", which finds its fullest form in the Karl Popper's works, and on the other hand, the "alternative perspective", which is expressed in the work of Boaventura de Souza Santos. The text argues that the field in which the cognitive foundations are settled is more political than epistemological.

Keywords: Epistemology - Karl Popper - Boaventura de Souza Santos "Hegemonic perspective" - "Alternative perspective" -

En este texto se comparan los principales lineamientos de la obra de Karl Popper con aspectos centrales del pensamiento epistemológico de Boaventura de Sousa Santos, tomados de su libro "Una Epistemología del Sur: la reinvención del conocimiento y la emancipación social". A partir de la posición de cada uno de estos dos autores contrastamos dos grandes cosmovisiones respecto de la ciencia, y acaso no sólo de la ciencia sino incluso del conocimiento humano contemporáneo en sentido amplio. Se trata de cosmovisiones, no de escuelas teóricas; ni siquiera de posiciones epistemológicas sistematizadas y definidas porque dentro de cada uno de estos dos universos epistemológicos pueden ubicarse más de una perspectiva teórica con muy variados matices o, incluso aún, con diferencias importantes.

Por el lado de Karl Popper, se erige una modalidad de concebir el conocimiento científico que aquí se llamará, genéricamente, "Perspectiva Hegemónica". Y desde el lugar de Boaventura de Sousa Santos aparece una modalidad que en estas páginas se denominará, también de manera general, "Perspectiva Alternativa" del conocimiento científico. Queda claro que la contrastación de ambos "universos epistemológicos" cobra sentido en la medida de que se trata de cosmovisiones distintas pero sobre todo enfrentadas. En efecto, aquí se sostiene que la principal oposición entre ambas perspectivas cognoscitivas se debe más bien a un enfrentamiento ideológico donde se disputan posiciones políticas, que a diferencias ubicadas en el plano epistemológico en sentido estricto. Dicho de otra manera, lo que importa en esta disputa son las consecuencias políticas de la misma; ya que una posición aparece como funcional a los intereses hegemónicos de los grupos sociales, países y regiones "dominantes", y la otra a los intereses de los grupos sociales, países y regiones "dominados". Cabe destacar la importancia que la variable "territorio" cobra en este tema, puesto que dentro de esta disputa epistemológica por un lado, se agruparían los intereses cercanos a las regiones del norte (destacándose aquí Europa y los Estados Unidos) y por otro lado, los intereses postergados de las regiones del sur (donde resalta Latinoamérica).

\section{Karl Popper. "Perspectiva Hegemónica" del conocimiento científico.}

Cómo se ha dicho en los párrafos anteriores, bajo la denominación "Perspectiva Hegemónica" es posible congregar una gran variedad de posturas epistemológicas que se abren en un abanico temático que va desde pequeños matices diferenciales hasta importantes divergencias. No obstante, es posible agruparlas a todas en un mismo universo epistemológico porque todas ellas refieren a una forma hegemónica que domina la concepción actual de la ciencia y que se sostiene desde las "grandes universidades", y desde los centros de conocimiento e investigación "consagrados" de los países centrales. Además, a partir de la globalización económica, cultural y política, esta concepción 


\section{Papeles de Trabajo No 33 - Julio 2017 - ISSN 1852-4508 \\ Centro de Estudios Interdisciplinarios en Etnolingüística y Antropología Socio-Cultural}

dominante se ha expandido mundialmente auto presentándose a su vez cómo la única manera válida de concebir el conocimiento. No sólo el científico sino el conocimiento humano en general; porque este "universo" identifica a la ciencia cómo la única forma de conocer socialmente legitimada, invisibilizando a toda otra tradición del saber. Tal posicionamiento cognoscitivo inicialmente identificado con el "positivismo" hoy día, cómo se muestra en este artículo, ha tomado más bien la forma de "racionalismo crítico". Esto es, según veremos, la postura planteada por Karl Popper.

A partir de 1934, con la publicación de "La lógica de la investigación científica, Popper introdujo algunos cambios importantes en la manera de pensar la forma en que opera la ciencia. Estos cambios, sin embargo, de ninguna manera rompieron con el fondo de los postulados de la "Perspectiva Hegemónica", "visión clásica moderna" o "arquetípica" de concebir a la ciencia. Por el contrario, como si se siguiera la máxima "cambiar algo para que nada cambie", a ellos se debe su vigorosa subsistencia en nuestros días.

Preocupado por las deficiencias más evidentes del modelo científico clásico, en especial la imposibilidad lógica de probar las teorías a través de la verificación ${ }^{2}$; Popper idea un método falsacionista el cual, aunque igualmente incapaz de verificar, si sería capaz de falsar. A partir de este método se podría demostrar de manera segura la falsedad de un enunciado científico, y de esta manera sería posible descartar definitivamente a las teorías "erradas" y mantener de manera provisoria a las que resistieron los intentos de falsación. No sólo la ciencia sería "salvada", sino las posibilidades últimas de la razón humana y por tanto todo el proyecto moderno.

Popper sostenía que la ciencia es un producto humano cuyo fin es resolver problemas a través del método de "ensayo y error". Dentro de tal método proponía que no había que entusiasmarse demasiado con los ensayos acertados; había que tomarlos como éxitos relativos y aceptarlos sólo provisoriamente; pues escapa a las posibilidades humanas saber "cómo son las cosas". En cambio a los ensayos fallidos había que descartarlos definitivamente, pues sí está dentro de las posibilidades humanas saber "cómo las cosas no son". Y por eso mismo era posible entusiasmarse con tales ensayos fallidos; porque a través del descarte seguro de los errores, a través de saber "cómo las cosas no son", es posible acercarse cada vez más a lo que se busca. La única condición es mantenerse en movimiento.

Resumiendo la postura, puede afirmarse que el falsacionismo de Popper, lo que hace, es desplazar las posibilidades de la razón humana, de la instancia de la construcción del conocimiento científico (como hacían los primeros positivistas) a la instancia del control del mismo. La fórmula lógica de éste planteo es la argumentación del modus tollen. Que establece, que si bien es imposible establecer generalizaciones universales a través de un número finito de observaciones particulares, si es posible, en cambio, a partir de un sólo hecho particular establecer la falsedad de la generalización. Dicho de otra manera, las

\footnotetext{
${ }^{2}$ La verificación pretende establecer la "verdad" de un enunciado a través de un razonamiento de corte inductivo. En el sentido de que si un acontecimiento se observa repetidas veces es posible establecer a partir del mismo un principio general.
} 


\section{Papeles de Trabajo No 33 - Julio 2017 - ISSN 1852-4508 \\ Centro de Estudios Interdisciplinarios en Etnolingüística y Antropología Socio-Cultural}

posibilidades de la ciencia no están en la construcción del conocimiento sino en su control crítico. De aquí el nombre de "racionalismo crítico" con que se conoce su escuela.

Como ya dijimos, existen sin dudas, diferencias entre la visión de Popper y la postura decimonónica positivista que conformó el modelo clásico de ciencia moderna, la cual planteaba un modelo aún más fuerte de ciencia al sostener la verificación en lugar de la falsación como criterio de justificación. Pero en lo que atañe a sus alcances últimos y, lo que termina siendo más relevante, en lo que hace a las implicancias políticas de ambas visiones, resulta claro que la postura popperiana se ubica dentro de la "Perspectiva Hegemónica" de la ciencia junto con el positivismo clásico del cual en última instancia no es sino su versión más fundamentada.

Si bien el modelo de Popper es más modesto en sus pretensiones al negar la posibilidad de la verificación, se trata también de una concepción de ciencia en extremo fuerte ${ }^{3}$ y mantiene el grueso de los supuestos básicos positivistas; en especial la confianza casi ciega en un modelo único de razón. Acaso el punto que implica la mayor continuidad entre el positivismo y el racionalismo crítico es que, tanto para la ciencia clásica como para el falsacionismo popperiano, el sujeto que conoce no tiene un papel decisorio en el proceso de conocer. La ciencia clásica objetivista, sostenía una noción de verdad especular, donde el objeto era aprendido pasivamente por el sujeto; y en la postura popperiana si bien el sujeto tiene preponderancia en el contexto de producción del conocimiento no lo tiene en el contexto de justificación del mismo, que cómo ya se ha visto, es el fundamental o clave en el modelo del racionalismo crítico. El propio Popper (Popper: 1994) explicita que la suya es "una epistemología sin sujeto cognoscente".

En suma, la "perspectiva hegemónica" el proyecto moderno positivista de la ciencia clásica, aquel que encontró su germen en la Grecia antigua y se lanzó a conquistar el mundo a partir de finales del siglo XVI, supo ser salvado gracias al racionalismo crítico. Karl Popper estaría, sin lugar a dudas, orgulloso de ello,

La economía capitalista y la ideología política liberal, sus grandes impulsores, siguen hoy, cómo ayer, apoyados y apoyándose en este modelo de ciencia; formando parte indisoluble de una manera particular de comprender y relacionarse con el mundo: la modernidad.

\section{Boaventura de Sousa Santos. "Perspectiva Alternativa" del conocimiento científico.}

El otro universo epistemológico en el cuál, se insiste, coexisten también varias posiciones epistemológicas y no una sola; propone un modelo alternativo y por lo tanto crítico a la

\footnotetext{
${ }^{3} \mathrm{El}$ falsacionismo supone la posibilidad de negar las teorías de forma absolutamente segura y objetiva. A través de la crítica racional al conocimiento, que presupone una correcta utilización (método único) de la razón humana (concebida a la manera de la ciencia clásica como universal), es posible conocer el mundo (si bien por vía negativa) y decidir objetivamente por la mejor de las teorías.
} 


\section{Papeles de Trabajo No 33 - Julio 2017 - ISSN 1852-4508 \\ Centro de Estudios Interdisciplinarios en Etnolingüística y Antropología Socio-Cultural}

"Perspectiva Hegemónica". En esta gran cosmovisión se ubica en un lugar destacado el pensamiento de Boaventura de Sousa Santos.

Explícitamente interesado en intervenir en la realidad social contemporánea donde identifica grupos sociales, pueblos, países y regiones oprimidos que no gozan de los mismos estándares de bienestar que otros grupos; Boaventura de Sousa Santos plantea que estas diferencias comienzan, y se sostienen, desde una cosmovisión particular del saber que en los términos del presente texto sería la "perspectiva hegemónica" de la ciencia. En tal sentido, el autor sostiene que el problema de las desigualdades no es tanto una cuestión de justicia social sino de "justicia cognitiva". Es que los saberes de ninguna manera son neutros como proclama la "perspectiva hegemónica". No existe algo así como una racionalidad "inocente". Toda epistemología tiene implícita o explícitamente una posición política que deviene de una particular forma de ver el mundo. Es en tal sentido, que Santos, propone su "Epistemología del Sur": un enfoque alternativo de la realidad compuesto por las ideas, saberes, cosmovisiones, concepciones del mundo, etc. que reconoce el saber producido desde las sociedades oprimidas por los intereses de los grupos hegemónicos: el capitalismo desregulado, el neo colonialismo; el patriarcado, etc. La "Epistemología del Sur" supone un pensamiento situado, es decir que reconozca sus intereses regionales y también su condición de opresión; porque el "Sur" no sólo es una geografía sino una "metáfora del sufrimiento".

El autor propone la existencia de un conocimiento alternativo y crítico, que se origina en los pueblos y especialmente en los pueblos organizados, y en los movimientos sociales. Estos producen un saber no científico que no proviene de los centros de conocimientos consagrados internacionalmente ni de las universidades, pero que resulta muy importante para reconocer las realidades propias de un territorio o grupo oprimido, alcanzar el bienestar, la dignidad, la cohesión social, mantener la biodiversidad, etc. Una vez visibilizado este saber y reconocido como valioso, aunque no sea científico, hay que ponerlo en contacto con el saber producido en las universidades.

El diálogo entre saberes que propone la "Epistemología del Sur" supone una apertura de racionalidades. Habría que pasar de un único modelo de razón a un modelo pluralista; una razón cosmopolita, a este cambio Santos lo llama "ecología del saber" lo que implica la incorporación en las universidades de otros conocimientos distintos del científico.

La ecología del saber que une a la universidad (y otros centros de conocimientos científicos tales como organismos de investigación científica y técnica) con los movimientos sociales para producir un pensamiento crítico, no solamente favorecería a los movimientos sociales sino también a tales centros de conocimiento. De esta manera, nace un conocimiento cualitativamente más calificado para enseñar y aprender; un conocimiento más amplio y por sobre todo un conocimiento crítico capaz de modificar la situación de los sectores sociales oprimidos atendiendo a sus situaciones y realidades específicas. De tal modo, se pueden discutir por ejemplo, en un dialogo entre la universidad y otros organismos productores de conocimiento científico, y el pueblo (movimientos sociales), cuestiones tales como la opresión latinoamericana bajo la égida de los organismos internacionales económicos, el neo liberalismo, el modelo extractivista, el estado, la democracia, la biodiversidad, etc. 


\section{Papeles de Trabajo No 33 - Julio 2017 - ISSN 1852-4508 \\ Centro de Estudios Interdisciplinarios en Etnolingüística y Antropología Socio-Cultural}

Por otro lado, en su alianza con los movimientos sociales, la universidad pública podría mantener su función emancipadora pudiendo resistir a la desinversión estatal frente a un estado preocupado únicamente en invertir en las áreas con fuerte interés de mercado (biología, química, ingenierías, etc.) y a la competencia de las universidades privadas y a la globalización socioeconómica de las ideas e intereses de los países centrales.

En definitiva, la propuesta de Boaventura de Souza Santos es dejar de lado el paradigma de la ciencia moderna; lo que en este artículo denominamos "Perspectiva Hegemónica" del conocimiento científico. El cambio de paradigma no es irracional, ni supone un mero escepticismo al estilo "posmoderno"; por el contrario la implementación de una "Epistemología del Sur" o, en términos de este trabajo, de una "Perspectiva Alternativa" supone un optimismo más fundado y una racionalidad más plural.

La ciencia moderna es, para Santos, excluyente y totalitaria y produce un conocimiento ambicioso que en última instancia genera desigualdad. Su propuesta en cambio es "un conocimiento prudente para una vida decente" (.p. 40).

En su libro "Una Epistemología del Sur: la reinvención del conocimiento y la emancipación social", Santos establece que para cambiar el paradigma tradicional de la ciencia moderna hay que superar 4 grandes dicotomías:1) ciencias naturales y ciencias sociales; 2) conocimiento local / conocimiento total; 3) sujeto / objeto del conocimiento; 5) Conocimiento científico y conocimiento común. También plantea la necesidad de construir un pensamiento que no delimite fronteras rígidas. Es que la ciencia moderna supone un pensamiento abismal ya que traza una línea divisoria que delimita lo existente de lo no existente. En efecto, desde su racionalidad única y a través de las universidades y organismos de investigación que detentan el monopolio de la producción del conocimiento, la ciencia genera un espacio racional y civilizado, dejando por otro lado un espacio irracional e incivilizado que se identifica con los conocimientos no científicos, pero también con lugares "bárbaros"; con topus peligrosos al estilo del estado de naturaleza hobbesiano. Esta división excluyente de fronteras se fortalece de la mano de la globalización neoliberal conformando un nuevo colonialismo, pero además también genera otras formas de opresión social y de "injusticias cognitivas". Boaventura de Souza Santos propone en cambio practicar un pensamiento superador de estos espacios abismales al que llama "pensamiento pos abismal". Se trata de una ecología de los saberes donde se dialoga entre las distintas formas de conocimiento y no se establecen discriminaciones entre diversos topus.

La posición epistemología de Santos es por todo lo visto pluralista pero no relativista; no demoniza el conocimiento científico, ni los logros de una razón instrumental; pero postula una razón cosmopolita, abocada a lograr la emancipación social.

\section{Resumen de dos cosmovisiones del conocimiento}


Antes de trazar algunas reflexiones críticas respecto a estos dos universos cognoscitivos, se ofrece, a manera de resumen, un cuadro donde se destacan de manera contrapuesta algunos de sus principales aspectos bajo la forma que toman en Karl Popper y Boaventura de Souza Santos.

\begin{tabular}{|c|c|}
\hline $\begin{array}{c}\text { Perspectiva Hegemónica del } \\
\text { Conocimiento } \\
\text { (Ejemplo: Karl Popper) }\end{array}$ & $\begin{array}{c}\text { Perspectiva Alternativa del } \\
\text { Conocimiento } \\
\text { (Ejemplo: Boaventura de Souza } \\
\text { Santos) }\end{array}$ \\
\hline Racionalismo crítico & Epistemologías del sur \\
\hline $\begin{array}{l}\text { Ciencia tradicional } \quad \text { (producida } \\
\text { monopólicamente en las universidades) }\end{array}$ & $\begin{array}{l}\text { Ecología de los saberes (incorporación de } \\
\text { otros saberes -diálogo entre ciencia y } \\
\text { movimientos sociales) }\end{array}$ \\
\hline Razón universal (modelo único) & Razón pluralista (modelo cosmopolita) \\
\hline $\begin{array}{l}\text { Razón “inocente" (pretensión de } \\
\text { neutralidad) }\end{array}$ & $\begin{array}{l}\text { Teoría crítica y pensamiento situado } \\
\text { (toma de partido) }\end{array}$ \\
\hline Razón instrumental (tecno ciencia) & $\begin{array}{l}\text { Razón emancipadora (ciencia de la vida } \\
\text { buena) }\end{array}$ \\
\hline Ciencia excluyente & Ciencia inclusiva \\
\hline $\begin{array}{l}\text { Modelo identificado con el norte (Europa } \\
\text { y los Estados Unidos) }\end{array}$ & $\begin{array}{l}\text { Modelo identificado con el sur } \\
\text { (Latinoamérica y los países, grupos y } \\
\text { regiones oprimidos) }\end{array}$ \\
\hline $\begin{array}{l}\text { Modelo de universidad globalizada y } \\
\text { orientada a los mercados trasnacionales }\end{array}$ & $\begin{array}{l}\text { Modelo de universidad pública orientada } \\
\text { a los movimientos sociales regionales }\end{array}$ \\
\hline
\end{tabular}

\section{Conclusiones}

El valor epistémico del cuestionamiento de los postulados del modelo moderno tradicional, hegemónico de ciencia reside en su sentido crítico. La "crítica" no implica aquí, de ningún modo, una impugnación en bloque. No se trata de desconocer la enorme revolución que produjo, a partir de la modernidad, el conocimiento científico con resultados espectaculares que configuraron una civilización occidental con estándares de bienestar y posibilidades jamás antes vistos, y además, una civilización liberadora para el ser humano en más de un sentido. No es la idea de la crítica, entonces, demonizar a la ciencia. El sentido de la crítica es, en este caso, analizar el modelo hegemónico del 


\section{Papeles de Trabajo No 33 - Julio 2017 - ISSN 1852-4508 \\ Centro de Estudios Interdisciplinarios en Etnolingüística y Antropología Socio-Cultural}

conocimiento para, a través de la aplicación de una suerte de cuñas teóricas, poner de manifiesto los postulados en los que descansa, y las implicancias que esto conlleva. Su función es sacar a la luz lo que ocultamente subyace en tal concepción del conocimiento $\mathrm{y}$, luego, proponer alternativas.

Resulta claro entonces, que la ciencia hegemónica que actualmente se auto adjudica excelencia epistemológica, y neutralidad valorativa; o lo que Heler llama garantía de verdad y objetividad (Heler: 2009) contiene varios di |lemas. El primero, quizá, es su auto adjudicación de "cientificidad" y "objetividad". Concepción excluyente bajo la cual se desestima toda otra forma de saber, y con ello, no admite ningún modo de juzgamiento. La ciencia, entonces, se aísla de la sociedad y se transforma en un bloque totalitario que no se deja cuestionar; en palabras de Santos se torna "totalitaria y excluyente". También es débil la idea de excelencia epistemológica; tanto por los límites que han demostrado tener sus distintos abordajes teóricos y metodológicos como incluso por sus propios resultados que pueden fácilmente cuestionarse desde perspectivas varias. Mucho más incierto aún, es el postulado que establece la "neutralidad valorativa". Resulta evidente que la ciencia se encuentra, a través de la utilización de la razón instrumental, interviniendo en todo el complejo entrazado de interacciones humanas de intercambio para producir y reproducir la vida económica y social. En este sentido, es más exacto hablar de una "tecno ciencia" que opera en todas los aspectos de la vida social, y que se encuentra estrechamente ligada a los mercados capitalistas concentrados. Lo que implícitamente la lleva a responder a los intereses de las regiones más ricas o regiones del Norte. En tal sentido la hegemonía que, a través del monopolio universitario y de los centros de producción de conocimientos canónicos, poseen los países centrales, que exportan de la mano de la globalización socio cultural sus intereses, y controlan la distribución del saber repartiendo incentivos tales como poder, prestigio y dinero, de ninguna manera significa neutralidad.

Una de las inquietantes cuestiones que se desprende de estos puntos es si la ciencia hoy, al margen de las distintas disciplinas, es un único producto que responde a un proyecto hegemónico con lógicas implícitas de las que no es posible desprenderse más allá de los matices. Dentro de este marco que no parece ser demasiado flexible ¿Cuál es el papel de las ciencias sociales? ¿Tienen un espacio de libertad mayor en cuanto a su fundamentación epistémica? ¿Tienen la posibilidad de resignificar a las otras ciencias? ¿Pueden responder a un proyecto que suponga una cosmovisión plural? ¿O más bien participan del modelo común de las ciencias físico-naturales? ¿Hasta qué punto? ¿Con qué poder?

Sin impugnar en bloque el modelo de ciencia predominante, debería estar claro, para quienes pretenden un cambio social en el sentido de una equiparación de los estándares de vida entre las distintas regiones, y un desarrollo tecnológico racional que contemple a la biodiversidad y la sustentación de la humanidad en el tiempo, que es indispensable una apertura del conocimiento científico. Una apertura hacia otras tradiciones de conocimiento; una racionalidad pluralista y el inicio de un diálogo con distintos grupos

\footnotetext{
${ }^{4}$ Por tecno ciencia se entiende la fusión de la ciencia y la tecnología en el mundo contemporáneo donde la ciencia aparece supeditada a su dimensión instrumental de acuerdo a las pautas regidas por la lógica capitalista.
} 
sociales que alejaría a la ciencia de esta posición totalitaria y excluyente. Para todo esto es indispensable, especialmente para las regiones oprimidas, revisar cuestiones como la financiación de la investigación científica y las posibilidades de lograr un sistema de producción del conocimiento abierto, sometido al control social, que sea consciente de sus intereses regionales y capaz de escapar a la lógica productiva del mercado donde la distribución de incentivos y posiciones de poder entre sus integrantes está regida en última instancia por el interés de la producción en el sentido de la ganancia.

Para todo ello es indispensable examinar el papel de los organismos de investigación y de conocimiento, y el de las universidades públicas; donde además de la producción de un saber enriquecido por una pluralidad de razones, éstos se encuentren comprometidos con la realidad social de la cual forman parte.

Este podría ser el papel del conocimiento del siglo XXI, y Latinoamérica, acaso pudiera tener un protagonismo inédito en la historia del conocimiento moderno.

\section{Bibliografía}

HELER, M., Ciencia incierta. Buenos Aires. Biblos.2009

POPPER, Karl: Sociedad abierta, universo abierto, Tecnos, Madrid, 1992.

POPPER, Karl: En busca de un mundo mejor, Paidós, Barcelona, 1984.

POPPER, Karl: La lógica de la investigación científica, Tecnos, Madrid, 1982.

POPPER, Karl: Conjeturas y refutaciones, Paidós, Bs. As., 1973.

POPPER, Karl: Conocimiento objetivo, Madrid, Tecnos, 1974.

POPPER, Karl: Búsqueda sin término, Tecnos, Madrid, 1985.

POPPER, Karl: La lógica de las ciencias sociales, Grijalbo, México, 1985.

SANTOS, Boaventura de Souza: Una epistemología del sur: la reinvención del conocimiento y la emancipación social, Siglo XXI, CLACSO, México.

VÁZQUEZ, Amancio: “Karl Popper y Claude Lévi-Strauss. Convergencias y Analogías”, Memorias del 53 Congreso ICA, México, 2009. 